Archived version from NCDOCKS Institutional Repository http://libres.uncg.edu/ir/asu/

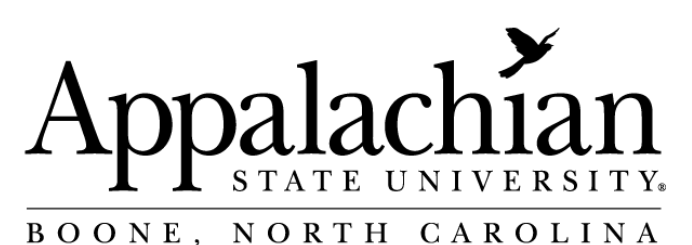

\title{
Imaging Emission Spectra With Handheld And Cellphone Cameras
}

\author{
By: David Sitar
}

\begin{abstract}
As point-and-shoot digital camera technology advances it is becoming easier to image spectra in a laboratory setting on a shoestring budget and get immediate results. With this in mind, I wanted to test three cameras to see how their results would differ. Two undergraduate physics students and I used one handheld 7.1 megapixel (MP) digital Cannon point-and-shoot auto focusing camera and two different cellphone cameras: one at $6.1 \mathrm{MP}$ and the other at $5.1 \mathrm{MP}$.
\end{abstract}

Sitar, D. (2012). "Imaging Emission Spectra with Handheld and Cellphone Cameras." The Physics Teacher 50(9): 524-525. https://doi.org/10.1119/1.4767480. Publisher version of record available at: https://aapt.scitation.org/doi/ full/10.1119/1.4767480 


\section{Imaging Emission Spectra with Handheld and Cellphone Cameras}

David Sitar, Appalachian State University, Boone, NC

A s point-and-shoot digital camera technology advances, it is becoming easier to image spectra in a laboratory setting on a shoestring budget and get immediate results. With this in mind, I wanted to test three cameras to see how their results would differ. Two undergraduate physics students and I used one handheld 7.1 megapixel (MP) digital Cannon point-and-shoot auto focusing camera and two different cellphone cameras: one at 6.1 MP and the other at 5.1 MP.

\section{Method}

Using each of the cameras and a Paton Hawksley 600 lines/ $\mathrm{mm}$ transmission grating held in front of the lens, a picture with no flash was taken from a distance of $1 \mathrm{~m}$ away of four different $4.75-\mathrm{cm}$ light-emitting spectral tubes. Hydrogen, helium, neon, and argon were excited by a $1800-\mathrm{V}$ power supply and, while illuminated, images were taken of the first-order $(n)$ emission lines.

\section{Results}

The top images of the following four sets of figures are from the Cannon handheld camera and the next group of images are from an online resource that we used to compare our results to. ${ }^{1}$ The only image manipulation done was some cropping and resizing in a word processor in order for them to fit in these columns; otherwise they are raw images. Therefore, they are not to scale to what you might get. You will also notice that each of the wavelength axes do not line up with the published spectra; this is due to the red and blue/violet sensitivity of the camera, which does not represent the "true" color scale or pixel ratio of the online resources wavelengths. We did not post any of the cellphone images, but the one with the 6.1 MP was quite similar to these.
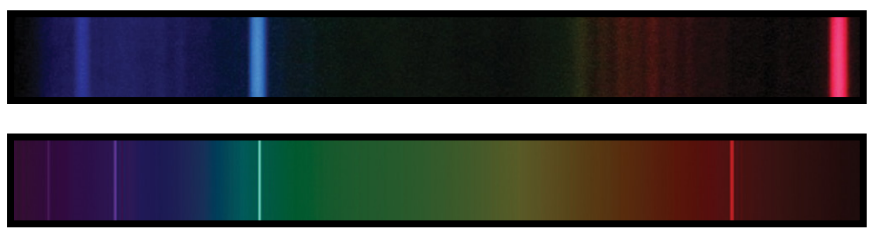

Fig. 1. Hydrogen.
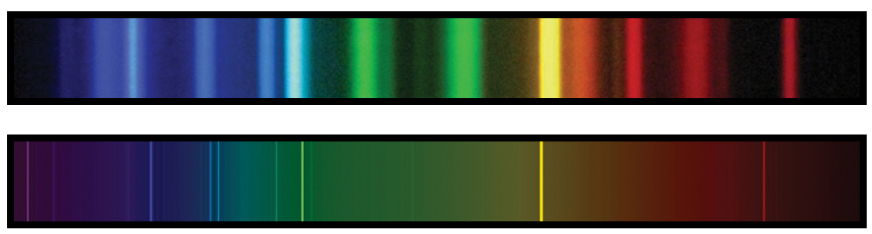

Fig. 2. Helium.

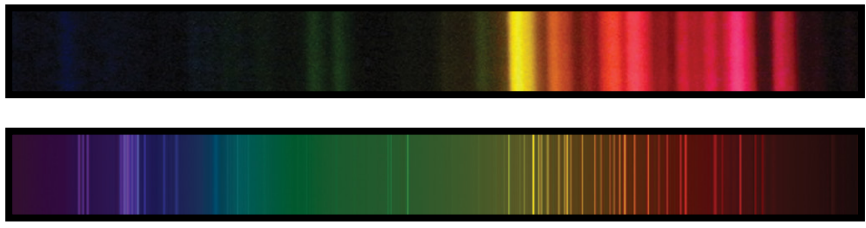

Fig. 3. Neon.

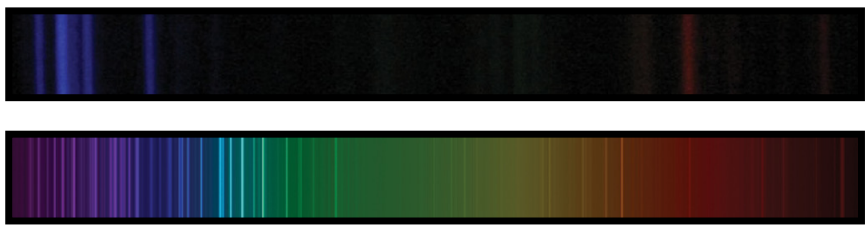

Fig. 4. Argon.

\section{Improvements}

Imaging spectra with handheld and cellphone digital cameras was successful, but ones with greater than $6 \mathrm{MP}$ produced the best results. What we did find in helping with the resolution of our pictures was to increase brightness of the spectral tubes. So, by using a $5000-\mathrm{V}$ power supply that held $26-\mathrm{cm}$ spectral tubes, we were able to achieve this, but it was not the equipment used to publish our results or by the students during their spectroscopy lab experience. If you do choose to use the $5000-\mathrm{V}$ power supplies, extra precautions must be taken for students' safety due to the exposed electrodes.

Last, experimenting with DSLR cameras, exposure times, manual focus, and the use of a tripod would definitely help with the image clarity. However, this method has repeatedly been done in physics labs and I was more interested to see if handheld devices such as ones that students may already have could provide adequate enough images to determine the element's signature.

\section{Extension}

Students who have had geometry or are in the process of taking it could, during the imaging process and with a few additional materials (such as a meterstick), measure the distance from the diffraction grating to the discharge tube $\left(d_{1}=1 \mathrm{~m}\right)$ and the distance $\left(d_{2}\right)$ from the tube to each of the emission lines $(\lambda)$, and solve for each $\left(d_{3}\right)$ and $(\theta)$. Then, with a calculator or pencil and paper, and the grating equation, $n \lambda=d \sin \theta$, wavelengths can be calculated and compared to accepted values. See Fig. 5 for details.

This calculation provides an opportunity for students to not just do a calculation and the necessary applied geometry, but also to perform unit analysis (and we just know how much our students love doing that) between angstroms $(\AA)$, 


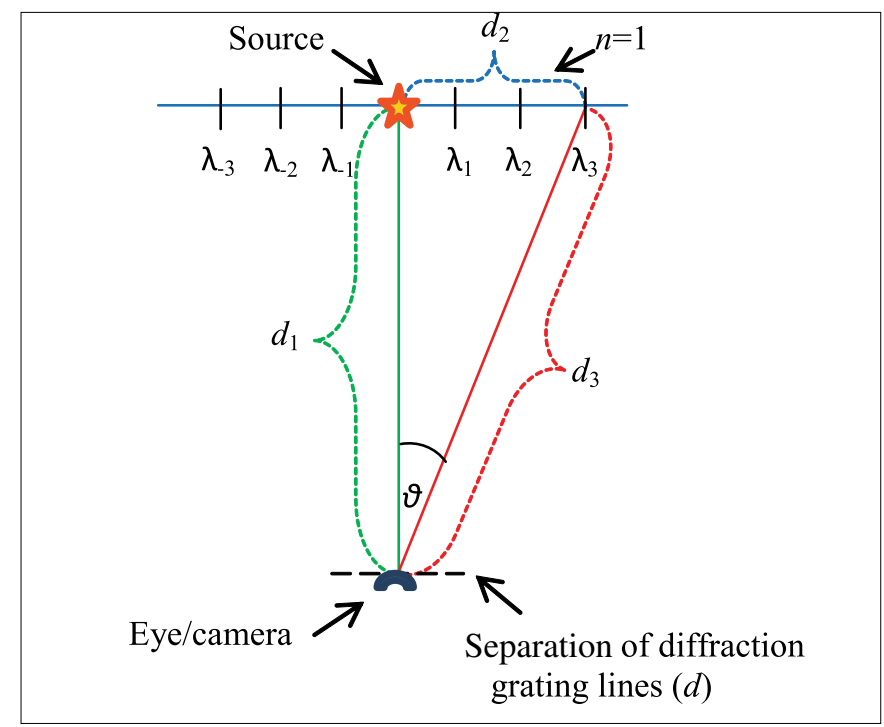

Fig. 5. Schematic.

nanometers $(\mathrm{nm})$, meters $(\mathrm{m})$, etc. Then, to cross disciplines, a discussion of the history of spectroscopy may be included in the lesson, as well as a writing assignment. To name a few topics, how did the Fraunhaufer spectrum come to be? Who was Andres Jonas Ångström? Or, what are Kirchhoff's three laws of spectral analysis?

\section{Comments}

Overall, I was quite impressed with the results and, even though the 5.1-MP cellphone camera produced rather fuzzy and unfocused images, it did give insight to what the lowest MP ratio that should be used is.

This is definitely something I am going to continue doing in my lab and, with some fine-tuning, I believe it will evolve into a great experiment and increase student learning.

If your lab, school, or department has the resources available, I recommend this lab experience. Students get to use their own cameras/cellphones, load their pictures to a computer or just use the camera's screen, and then compare their images to the online resource to see if they can deduce what the element is. ${ }^{1}$

\section{Acknowledgements}

I would like to thank the Department of Physics and Astronomy at Appalachian State University (ASU) for their support in this project. I would also like to thank Joe Pollock and Richard Gray for their input. Last, I would like to thank the two ASU physics undergraduates, Jeremy Bowen and Tim Gray, with their assistance in the data collection.

\section{Reference}

1. Spectra of gas discharge images, astro.u-strasbg.fr/ koppen/ discharge/index.html (Retrieved 4/5/2012).
David Sitar is an astronomy lab instructor at Appalachian State University. He has been teaching introductory astronomy courses and labs prior to and since receiving an MS from Eastern Michigan University in 2011. He is currently involved with public outreach, science education, teaching introductory astronomy labs, and developing lab curricula for the Rankin GoTo laboratory at ASU. sitardj@appstate.edu 